\title{
A health economic evaluation of needle-based confocal laser endomicroscopy for the diagnosis of pancreatic cysts
}

\section{(1) $\odot \odot$}

\author{
Authors \\ Claude Le Pen ${ }^{1}$, Laurent Palazzo², Bertrand Napoléon ${ }^{3}$ \\ Institutions \\ 1 Université Paris-Dauphine, Paris, France \\ 2 Clinique du Trocadéro, Paris, France \\ 3 Ramsay Générale de Santé, Hôpital Privé Jean Mermoz, \\ Lyon, France
}

submitted 22.2.2017

accepted after revision 4.7.2017

\author{
Bibliography \\ DOI https://doi.org/10.1055/s-0043-117947 | \\ Endoscopy International Open 2017; 05: E987-E995 \\ (c) Georg Thieme Verlag KG Stuttgart · New York \\ ISSN 2364-3722
}

Corresponding author

Claude Le Pen, Laboratoire d'Économie et de Gestion des

Organisations de Santé (LEGOS), Université Paris-Dauphine,

Place du Maréchal de Lattre de Tassigny, 75016 Paris,

France

Fax: +33-1-44054949

claude.lepen@dauphine.fr

\section{ABSTRACT}

Background and study aims The low sensitivity of endoscopic ultrasound-guided fine-needle aspiration (EUS-FNA), especially for the diagnosis of serous cystadenomas (SCAs), can be associated with diagnostic uncertainty that can regularly lead to unnecessary surgical procedures. Needlebased confocal laser endomicroscopy (nCLE) used with EUS-FNA improves diagnostic accuracy, helping to reduce unnecessary surgery and patient follow-up. This study was conducted to evaluate the economic benefit of EUS-FNA+ nCLE.

Patients and methods Probabilities used were derived from two studies representative of the two diagnostic strategies: a retrospective analysis of patients diagnosed by EUS-FNA alone and a prospective study of patients diagnosed by EUS-FNA+ nCLE. Costs were based on French healthcare system rates; both private and public sector rates were included. A decision tree structure model used these probabilities and costs for two hypothetical cohorts of 1000 patients.

Results EUS-FNA + nCLE resulted in a reduction of $23 \%$ in the total rate of surgical intervention, which translated to a reduction in clinical costs of $13 \%$ (public sector) and $14 \%$ (private sector). Additionally, the reduced rate of surgery would save the lives of 4 in 1000 patients. A stochastic sensitivity analysis using 100 simulations showed that in all cases the number of interventions was less for EUS-FNA+ $\mathrm{nCLE}$ than for EUS-FNA. There was also a reduction in the incidence of false negatives using EUS-FNA + nCLE.

Conclusions EUS-FNA + nCLE results in significant economic benefits by reducing the incidence of misdiagnosis through improved diagnostic accuracy.

\section{Introduction}

Pancreatic cysts are often detected during routine clinical examination, and their detection is increasing with more frequent use of cross-sectional imaging, e. g. during magnetic resonance imaging (MRI) procedures and computerized tomography (CT) scans $[1,2]$. Despite the recent advances in MRI procedures, differentiating the various types of cystic lesions is not always possible using this technique as there is both an overlap in morphological characteristics, making it difficult to predict malignancy, and poor inter-observer agreement with regard to the individual characteristics of pancreatic cysts [3]. While early detection of premalignant lesions is necessary to prevent the de- velopment of malignancy, many of these cysts are benign pseudocysts (PCs) and serous cystadenomas (SCAs) that do not require any treatment or follow-up.

In some cases, the clinical diagnosis can be highly suggestive (i.e. cysts that communicate with the pancreatic duct, suggestive of intraductal papillary mucinous neoplasms [IPMNs] or cysts combined with chronic calcified pancreatitis [CCP], suggestive of PCs). However, for an isolated, non-communicating cyst without CCP, the diagnosis can remain unclear after imaging procedures such as MRI and CT scans, and endoscopic ultrasound-guided fine-needle aspiration (EUS-FNA) is often considered. 
The EUS-FNA procedure, however, may not provide a definitive diagnosis due to the low sensitivity of cytology, especially for SCAs. Additional biochemical tests are used, such as carcinoembryonic antigen (CEA) and amylase dosage, but their specificity is too low to allow definite conclusions to be reached with regard to the nature of the cyst [4]. Moreover, cystic fluid CEA levels show considerable fluctuations over time in patients undergoing three or more EUS-FNAs [5]. Such diagnostic uncertainty in SCA-positive (SCA+) patients can result in unnecessary surgical intervention and regular, long-term surveillance, which creates an economic burden to healthcare resources as well as causing unnecessary physical and psychological trauma to the patient.

Needle-based confocal laser endomicroscopy (nCLE) used in conjunction with EUS-FNA (EUS-FNA $+\mathrm{nCLE}$ ) has recently been evaluated for the diagnosis of pancreatic cystic neoplasms [6, 7] and has shown excellent feasibility and safety, enabling a more confident diagnosis of SCA thanks to the visualization of a very specific pattern: the superficial vascular network [6]. This improvement in diagnostic accuracy, as well as the capacity to discriminate cysts that have the potential to become malignant (termed "suspect") from those that are benign, helps to reduce both unnecessary surgical intervention and long-term follow-up surveillance.

Currently there is no cost-effectiveness analysis of investigational tools to diagnose and follow up the progression of neoplastic cysts [8]. The aim of this study was therefore to evaluate the health economic benefit of the diagnosis of SCA with EUSFNA combined with nCLE rather than EUS-FNA alone, in the diagnosis and management of patients harboring an SCA, in terms of the overall cost of procedures and hospital stays as well as other associated healthcare costs.

\section{Materials and methods}

\section{Patients and surgical interventions}

We compared two hypothetical and identical cohorts of 1000 patients, each patient harboring an undetermined pancreatic cyst of at least $2 \mathrm{~cm}$ in diameter, without Wirsung duct communication and without CCP. The latter two criteria would be confirmed on cross-sectional imaging or during EUS imaging. The first cohort was referred for EUS-FNA (alone) and the second for EUS-FNA + nCLE. They were deemed to be statistically identical in terms of the prevalence of cyst type, comparable to patients randomly assigned to a diagnostic procedure. The diagnostic performances and probabilities used in this analysis were derived primarily from a retrospective analysis of $2622 \mathrm{pa}$ tients who were diagnosed with an SCA lesion between 1990 and 2014 in 23 countries and 71 centers [9], and secondarily from a prospective three-center study conducted in France [6]. The hypotheses for which no evidence is cited were based on theoretical considerations and expert opinion. - Table 1 summarizes the assumptions.

Hypothesis for the a priori probability of being SCA+

The a priori probability of a pancreatic cyst being SCA+ constituted the basis of our model. A previous study [6,7] enrolled
- Table 1 Assumptions: a priori probability of being SCA+, diagnostic performance, and surgical intervention for EUS-FNA and EUS-FNA+ nCLE.

\begin{tabular}{|c|c|c|}
\hline & \multicolumn{2}{|c|}{ Diagnostic procedure } \\
\hline & EUS-FNA & EUS-FNA + nCLE \\
\hline A priori probability of being SCA+, \% & \multicolumn{2}{|c|}{43} \\
\hline \multicolumn{3}{|l|}{ Diagnostic performance } \\
\hline - Sensitivity of test (SCA+), \% & $20^{1}$ & $69^{2}$ \\
\hline - Specificity of test (SCA+), \% & $90^{1}$ & $100^{2}$ \\
\hline \multicolumn{3}{|l|}{ Surgical intervention } \\
\hline - Tested population (SCA+), \% & $47^{3}$ & $10^{3}$ \\
\hline - Tested population (SCA-), \% & $50^{4}$ & $50^{4}$ \\
\hline \multicolumn{3}{|c|}{$\begin{array}{l}{ }^{1} \text { From cytology (Belsley et al. [11], Le Borgne et al. [12], Maker et al. [13], } \\
\text { Müssle et al. [14], Thornton et al. [15], Thosani et al. [16]). } \\
2 \text { From superficial vascular network using nCLE (Napoléon et al. [6]). } \\
{ }^{3} \text { Calculated from Jais et al. [9]. } \\
{ }^{4} \text { Based on Jais et al. [9] in which the resection rate of any type of pancreatic } \\
\text { cyst lesion ranged from } 54 \% \text { to } 94 \% \text { during the period } 2010 \text { to } 2014 \text {. }\end{array}$} \\
\hline
\end{tabular}

patients with the criteria described above, and so was considered to be representative of the percentage of SCA lesions in our targeted population. This a priori prevalence was $43 \%$, which is in agreement with the estimations of Brugge et al. [10], who reported that, among non-communicating cystic neoplasms of the pancreas, SCAs (32-39\%), mucinous cystic neoplasms (10-45\%), and IPMNs (21-33\%) represent the majority of cases encountered in routine clinical practice.

\section{Hypotheses for diagnostic performance}

The marker performance for SCA diagnosis is limited [4] and cytology alone has been taken into account for the assessment of EUS-FNA. While cytology has high specificity, it exhibits low sensitivity, especially for the diagnosis of SCA [11 - 16]. The superficial vascular network that is observed using nCLE has been shown to have a higher sensitivity (69\%) and a specificity of $100 \%$ for the characterization of SCA [6]. A summary of these hypotheses for diagnostic performance of EUS-FNA (cytology) and EUS-FNA + nCLE (using the superficial vascular network) is presented in $\triangleright$ Table 1.

\section{Hypotheses for surgical intervention rules}

The exceptional risk of malignancy is not considered to be a reason for systematic surgery. Resection is performed worldwide due to symptoms or diagnostic uncertainty (inability to distinguish a serous cystic neoplasm from a mucinous lesion). Depending on cultural trends (e.g. surgical vs medical centers), some centers also select patients for surgery based on tumor size and growth rate $[9,17]$.

The rules for surgical intervention in SCA+ patients were based on data from the European Study Group on Cystic Tumors of the Pancreas [9]. Of the 2622 patients included, 1032 patients (39\%) had no surgical intervention. Of the remaining 1590 patients (61\%), most (1367 patients [86\%]) underwent 
- Table 2 Medical results using model for a hypothetical cohort of 1000 patients.

\begin{tabular}{|l|l|r|}
\hline & EUS-FNA (n=1000 $)$ & EUS-FNA+nCLE (n=1000 $)$ \\
\hline Number of patients with surgery & $495(49.5 \%)$ & $381(38 \%)$ \\
\hline " Among the "true SCA+" & $212(43 \%)$ & $96(25 \%)$ \\
\hline " Among the "true SCA-" & $283(57 \%)$ & $285(75 \%)$ \\
\hline Number of patients without surgery & $505(49.5 \%)$ & $619(62 \%)$ \\
\hline " Among the "true SCA+" & $218(43 \%)$ & $334(54 \%)$ \\
\hline " Among the "true SCA-" & $287(57 \%)$ & $285(46 \%)$ \\
\hline Surgical mortality & $17(3.4 \%)$ & $13(3.4 \%)$ \\
\hline $\begin{array}{l}\text { Data are number of patients (percentage of patients). } \\
\text { 1 Hypothetical population of } 1000 \text { patients. }\end{array}$ & & \\
\hline
\end{tabular}

- Table 3 Thresholds for sensitivity and specificity used for the stochastic analysis of sensitivity.

\begin{tabular}{|l|l|l|l|l|}
\hline & EUS-FNA & \multicolumn{2}{|l|}{ EUS-FNA + nCLE } \\
\hline & Point estimate & Distribution interval & Point estimate & Distribution interval \\
\hline Sensitivity & 0.2 & $0.10-0.30$ & 0.69 & $0.60-0.80$ \\
\hline Specificity & 0.9 & $0.80-1.00$ & 1.00 & $0.80-1.00$ \\
\hline
\end{tabular}

surgery in the first year after diagnosis. Of the 1590 patients who had a surgical intervention, 954 patients (60\%) underwent surgery due to diagnostic uncertainty, 366 patients (23\%) due to the presence of symptoms, 190 patients (12\%) due to the size of the tumor or its growth, and 80 patients (5\%) due to compression of an adjacent organ by the tumor.

For the model included in this study, therefore, the proportion of patients SCA + with surgery for a reason other than the presence of symptoms was $47 \%$ ([1590-366]/2622 patients) and this was reduced to $10 \%$ if those with surgery due to diagnostic uncertainty were also excluded ([1590-366-954]/ 2622) as would be the case using EUS-FNA + nCLE rather than EUS-FNA alone. The surgery in the $10 \%$ of patients diagnosed with SCA+ using EUS-FNA + nCLE is mainly due to clinical reasons other than the tumor itself, e.g. compression of the bile duct or splenic vessels.

As described by Fernandez-del Castillo et al. [18], the prevalence of pseudocysts was rare in a cohort of patients with undetermined pancreatic cysts without Wirsung duct communication, without CCP, and referred for EUS-FNA. As such, the SCAnegative (SCA-) cohort would essentially be composed of patients with mucinous lesions (mucinous cystic neoplasms [MCN] and IPMN), with the incidence of MCN likely to be higher than IPMN since the cysts do not communicate. It is reasonable to consider that $50 \%$ of this SCA- cohort would be referred for surgery and $50 \%$ would undergo surveillance (this was the ratio in a previous study [6] in which $9 / 20$ patients were referred for surgery). Therefore, the proportion for surgical intervention in SCA- patients based on experts' experience was set at $50 \%$.

\section{Procedures for surgical intervention}

Duodenopancreatectomy (Whipple's surgery) (DP) or left pancreatectomy (LP) are offered to patients requiring a surgical intervention based on the rules described above. According to the French Technical Agency for Information on Hospitalization database (Agence Technique de I'Information sur l'Hospitalisation [ATIH]) [19], $80 \%$ of surgical interventions occur in the public sector and $20 \%$ in the private sector. The relative proportions of DP and LP are 80/20 in the public sector and 50/50 in the private sector. Of these, LP is a more straightforward procedure than DP as less pancreatic tissue is resected and there is no reconstructive component. As such, DP is undertaken mostly in the public sector.

A recent systematic review of outcomes of all pancreatic surgeries showed lower immediate postoperative mortality as well as long-term mortality for patients who undergo surgery in high-volume pancreatic centers (predominantly from centers of excellence) $[6,20]$. Thus, assuming a mortality rate of $4 \%$ for DP and $1.8 \%$ for LP, a global surgical mortality rate of $3.4 \%$ was calculated ( $\triangleright$ Table 2 ).

\section{Influence of test performance}

A stochastic analysis (Monte Carlo-type simulation) was performed to compare the influence of sensitivity and specificity of the two diagnostic methods, EUS-FNA + nCLE and EUS-FNA alone, on the final number of surgical interventions. The point estimates used in the model for EUS-FNA ( 0.20 for sensitivity and 0.90 for specificity) and EUS-FNA + nCLE (0.69 for sensitivity and 1.00 for specificity) were replaced by the uniform distribution intervals $(0.10-0.30$ and $0.80-1.00$ for EUS-FNA and $0.60-0.80$ and $0.80-1.00$ for EUS-FNA + nCLE, for sensitivity and specificity, respectively) ( $\triangleright$ Table 3$)[6,15,16]$. 


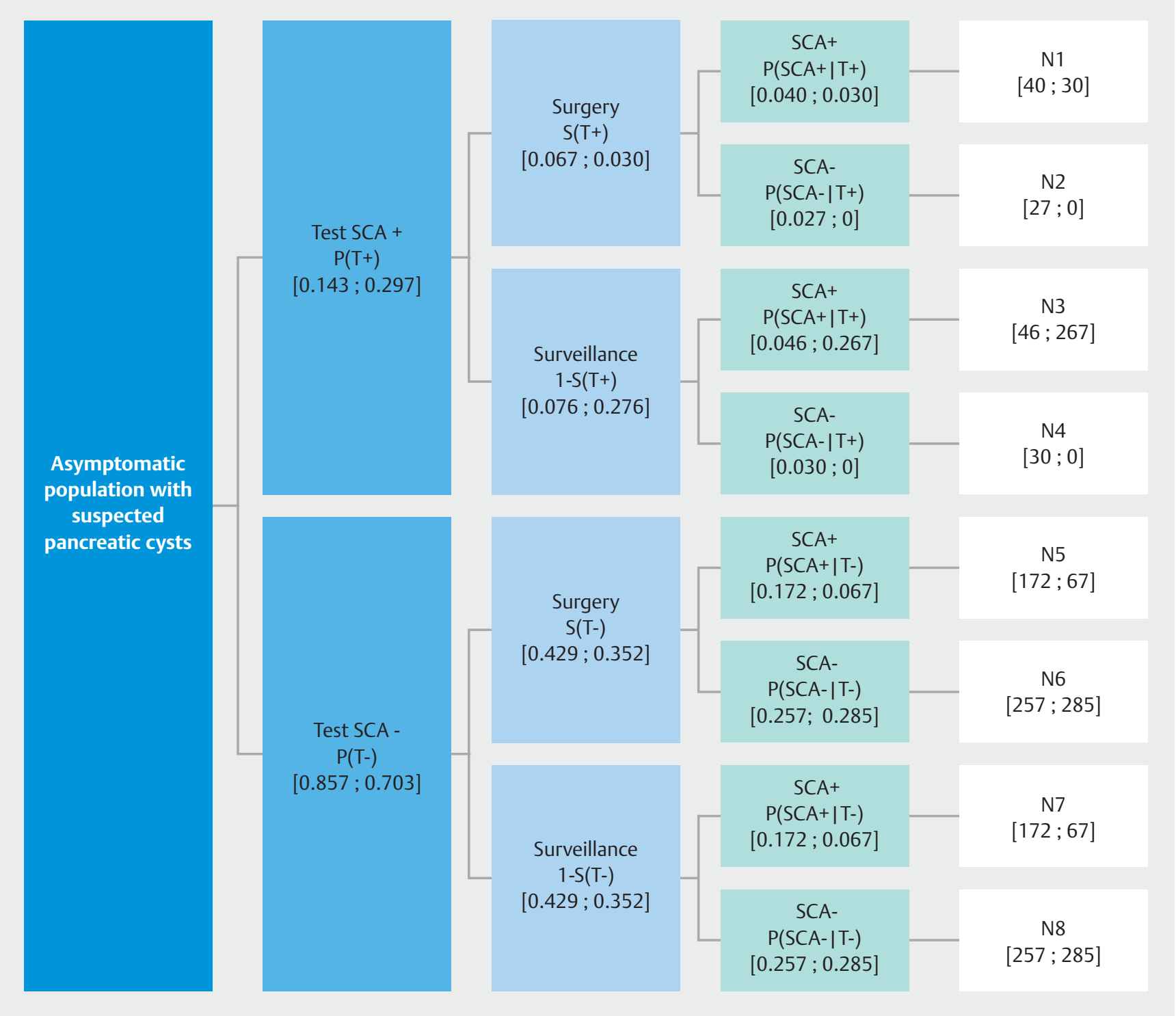

Fig. 1 Structure of model.

(Note: the same model is used for EUS-FNA and EUS-FNA + nCLE; the only differences are the probability values. Values indicated in brackets are the probability values for EUS-FNA and EUS-FNA $+n C L E$ displayed as the following [EUS-FNA; EUS-FNA + nCLE].)

Total number of surgical interventions: $\mathrm{N} 1+\mathrm{N} 2+\mathrm{N} 5+\mathrm{N} 6$.

Number of surgical interventions in patients tested SCA+: N1 + N2.

Number of surgical interventions in patients "true" SCA+: N1.

Number of surgical interventions in patients tested SCA-: N5 + N6.

Number of surgical interventions in patients "true" SCA-: N6.

\section{Analysis model}

A decision tree structure, adapted for probability analyses and diagnostic tests, was used. The same structure was used for the two groups (EUS-FNA + nCLE and EUS-FNA alone), and differed only in the probabilities used. The model consists of three main steps - these are described below and presented in > Fig. 1:

I. In the first step, asymptomatic patients diagnosed with a pancreatic cyst during routine examination are referred for a second examination to establish the type of lesion: these patients have a chance of presenting with a serous (benign:
$\mathrm{SCA}+$ ) lesion a priori, i.e. pre-test. The second diagnostic examination can be either EUS-FNA or EUS-FNA + nCLE, and identifies the lesion as either serous (benign: SCA+) or nonserous (suspect: SCA-). These a posteriori, or post-test, probabilities corresponding to SCA+ and SCA- are defined as $p(T+)$ and $p(T-)$, respectively.

II. In the second step, based on $p(T+)$ and $p(T-)$, a decision is then taken with regard to surgical intervention, marked as $\mathrm{S}(\mathrm{T}+)$ and $\mathrm{S}(\mathrm{T}-)$ for surgical intervention in SCA+ and SCApatients, respectively. Most non-serous (suspect: SCA-) lesions lead to surgical intervention irrespective of the method 
- Table 4 Total cost of diagnostic procedure and surgical intervention based on public and private sector rates for a hypothetical cohort of 1000 patients.

\begin{tabular}{|c|c|c|c|c|c|c|}
\hline & \multicolumn{3}{|c|}{ EUS-FNA } & \multicolumn{3}{|c|}{ EUS-FNA + nCLE } \\
\hline & $\mathbf{n}$ & Unit cost $(€)$ & Total cost $(€)$ & $\mathbf{n}$ & Unit $\operatorname{cost}{ }^{1}(€)$ & Total $\operatorname{cost}^{1}(€)$ \\
\hline \multicolumn{7}{|l|}{ Public sector } \\
\hline - Diagnostic procedure & 1000 & 337.55 & 337550.00 & 1000 & 937.55 & 937550.00 \\
\hline - Surgery in patients SCA+ & 212 & 14292.11 & 3029927.32 & 96 & 14292.11 & 1372042.56 \\
\hline - Surgery in patients SCA- & 283 & 16761.61 & 4743535.63 & 285 & 16761.61 & 4777058.85 \\
\hline Total & - & - & 8111012.95 & - & - & 7086651.41 \\
\hline \multicolumn{7}{|l|}{ Private sector } \\
\hline - Diagnostic procedure & 1000 & 337.55 & 337550.00 & 1000 & 937.55 & 937550.00 \\
\hline - Surgery in patients SCA+ & 212 & 10344.35 & 2193002.20 & 96 & 10344.35 & 993057.60 \\
\hline - Surgery in patients SCA- & 283 & 6290.34 & 1780166.22 & 285 & 6290.34 & 1792746.90 \\
\hline Total & - & - & 4310718.42 & - & - & 3723354.50 \\
\hline \multicolumn{7}{|c|}{$\begin{array}{l}\text { Data are based on two hypothetical populations of } 1000 \text { patients. } n=\text { number of patients. } \\
\text { Note: lower overall cost for surgical intervention in the private sector due to reduced hospital stay. } \\
{ }^{1} \text { The cost of nCLE is estimated at } 600 € \text {. }\end{array}$} \\
\hline
\end{tabular}

of diagnosis, with the rate fixed at $50 \%$ since some patients are inoperable. For serous (benign: SCA+) lesions, the surgical intervention rate depends on the method of diagnosis: for EUS-FNA $47 \%$ of patients undergo surgery, whereas for EUS-FNA $+n C L E$ surgery is required in only $10 \%$ of patients (as described above and in $>$ Table 1 ).

III. In the third step, the proportion of patients - among those who underwent surgery in the second step - who had a confirmed serous lesion (SCA+), and so would not have undergone surgery in an ideal diagnostic situation, is determined using Bayesian probability.

The second step uses probabilities of surgical intervention based on the result of the diagnostic test, whereas the third step uses the probability of being SCA+ based on having undergone surgery. Paired Student's t test was used to compare the number of surgical interventions of the two models, with $P<$ 0.05 considered to be significant.

\section{Economic hypotheses: cost of diagnostic procedures and surgical interventions}

Costs were estimated from the public payer's perspective, using official French rates in 2014, with no extra fees being paid by the patients.

For EUS-FNA, the cost was $274.87 €$ for the procedure plus $62.68 €$ for anesthesia, i.e. a total cost of $337.55 €$. As the EUS$\mathrm{FNA}+\mathrm{nCLE}$ medical device and/or procedure does not exist in the current nomenclature, the cost of nCLE was estimated at $600 €$ per patient (cost estimate provided by Mauna Kea Technologies), so the total cost of EUS-FNA + nCLE was estimated at $937.55 €$ ( $\triangleright$ Table 4).
The costs of surgery were $1021.74 €$ for DP plus $584.75 €$ for anesthesia, and $540.05 €$ for LP plus $355.28 €$ for anesthesia, i.e. total costs of $1606.49 €$ for DP and $895.33 €$ for LP.

Additional costs result from the hospital stay associated with a surgical intervention. Hospital stays are funded based on the GHS rate (Groupe Homogène de Séjours), which allows the stay to be allocated to a diagnostic related group (Groupe Homogène de Malades [GHM]). In France, these costs are classified separately for benign and malignant tumors with four levels for each class, dependent on the degree of complications. These costs are presented in $\mathbf{\sim}$ Table $\mathbf{5}$.

For public hospitals, the GHS rate is global and includes all of the charges associated with a patient's stay. For private hospitals and clinics, medical fees are billed in addition to the GHS rates based on the Joint Classification of Medical Procedures (Classification Commune des Actes Médicaux [CCAM]) nomenclature and rates, meaning that additional fees are added for the surgeon and anesthesiologist (1461.86€ and $1249.41 €$ for malignant and benign tumors, respectively).

\section{Results}

\section{Analysis of cost impact on patient management}

The interventions and status (true SCA+ and true SCA-) of a hypothetical cohort of 1000 patients based on the assumptions previously described for the model used are presented in $>\mathbf{T a}$ ble 2 .

The addition of nCLE to EUS-FNA allows a $23 \%$ reduction of surgical interventions for all patients (495 patients for EUSFNA and 381 for EUS-FNA + nCLE), and a $55 \%$ reduction for patients with benign tumors (the "true" SCA+ patients) (212 patients for EUS-FNA and 96 for EUS-FNA + nCLE) ( Table 2$)$. This reduction in surgical intervention results in a decrease in the 
- Table 5 Average hospital stay costs in France for DP and LP for benign and malignant tumors.

\begin{tabular}{|c|c|c|c|c|c|}
\hline \multirow[b]{2}{*}{ Tumor type } & \multirow[b]{2}{*}{ Level $^{1}$} & \multicolumn{2}{|l|}{ Public sector } & \multicolumn{2}{|c|}{ Private sector } \\
\hline & & Cost & Days $^{2}$ & Cost & Days $^{2}$ \\
\hline \multirow[t]{5}{*}{ Malignant } & 1 & $9613.24 €$ & 125 & $4125.08 €$ & 62 \\
\hline & 2 & $12762.69 €$ & 397 & $6347.99 €$ & 146 \\
\hline & 3 & $16321.80 €$ & 650 & $8341.35 €$ & 248 \\
\hline & 4 & $22674.00 €$ & 468 & $14711.72 €$ & 137 \\
\hline & Mean & $16761.61 €$ & & $8881.49 €$ & \\
\hline \multirow[t]{5}{*}{ Benign } & 1 & $6672.94 €$ & 35 & $2493.56 €$ & 3 \\
\hline & 2 & $9903.37 €$ & 74 & $3931.15 €$ & 20 \\
\hline & 3 & $14240.99 €$ & 66 & $5013.99 €$ & 23 \\
\hline & 4 & $29162.42 €$ & 40 & $8848.50 €$ & 8 \\
\hline & Mean & $14292.11 €$ & & $5040.99 €$ & \\
\hline \multicolumn{6}{|c|}{$\begin{array}{l}\text { DP, duodenopancreatectomy; LP, left pancreatectomy. } \\
\text { Note: These costs are derived from the French National Costs Scale based on information from a sample of public and private hospitals that annually report their } \\
\text { analytical costs for each diagnostic related group (GHM, French equivalent of the DRG). The costs are computed at patient discharge and may vary according to the } \\
\text { level of complications. The table shows costs for DRGs } 07 C 091 \text { to } 07 C 094 \text { for malignant tumors (i. e. liver, pancreas, portal vein or vena cava interventions for ma- } \\
\text { lignant tumors of Grade } 1 \text { to } 4 \text { ) and } 07 C 101 \text { to } 07 C 104 \text { for benign tumors (i. e. liver, pancreas, portal vein or vena cava interventions for benign tumors of Grade } 1 \text { to } \\
\text { 4). Fees are determined according to a national rate, apart from a large proportion of practitioners who are allowed to bill extra fees, generally covered by patients' } \\
\text { private health insurance on top of the mandatory public health insurance. In the final results for the private sector, fees from the national rate are therefore added to } \\
\text { the cost of the stay. } \\
1 \text { Based on increasing level of complications from surgery. } \\
2 \text { 'Days hospitalized due to DP or LP surgery. }\end{array}$} \\
\hline
\end{tabular}

overall cost of pancreatic cyst management, which exceeds the extra cost of the diagnostic technique, resulting in an economic benefit for both public and private sectors ( $>$ Table 4 ). Overall, the use of EUS-FNA $+n C L E$ results in a reduction of $13 \%$ (public sector) and $14 \%$ (private sector) in diagnostic and patient management costs (not including subsequent surveillance and treatment costs).

\section{Deterministic sensitivity analysis}

Influence of the a priori (pre-test) probability of presenting serous (benign: SCA+) cysts

This estimation is necessary for the calculation of the probability of "true" SCA+ patients who underwent surgery. The number of surgical interventions was calculated as a function of the a priori (pre-test) probability of a patient harboring a SCA. As described earlier, we used an a priori probability of $43 \%$ of a pancreatic cyst being SCA+, but it is important to evaluate the impact of this hypothesis on the results of the model and the final number of surgical interventions. A deterministic sensitivity analysis was therefore conducted on the [0.30-0.50] interval. Because of the lower specificity and the weak difference in surgery rates, the results in the EUS-FNA branch are not very sensitive to the variation of this parameter. However, this is no longer the case when nCLE is added. The number of surgical interventions using EUS-FNA + nCLE is inversely correlated to the a priori probability but remains inferior to that using EUS-FNA alone over the whole interval (see $>$ Fig. 2).

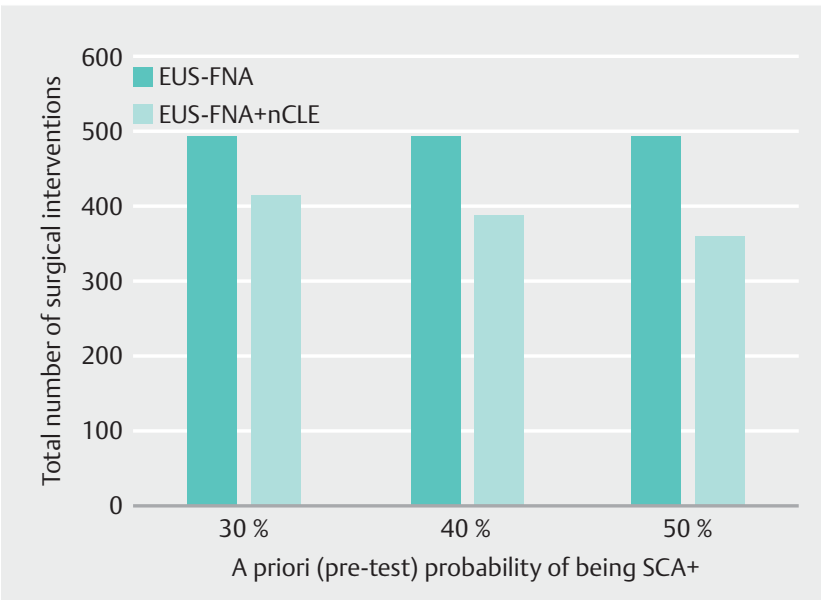

Fig. 2 Deterministic sensitivity analysis for the a priori probability of being SCA+.

Influence of the rules for surgical intervention using EUS-FNA + nCLE

As described earlier, the calculation has been performed based on the hypotheses of $47 \%$ (EUS-FNA group) and $10 \%$ (EUS-FNA $+n C L E$ group) of SCA+ patients being sent for surgery. Therefore, as the rate of detection of operated SCA+ patients using EUS-FNA + nCLE approaches that of using EUS-FNA alone, it is expected that the number of surgical interventions will converge. However, when considering a realistic rate of operated 
patients after diagnosis by EUS-FNA + nCLE, the number of surgical interventions remains significantly lower than that following diagnosis using EUS-FNA alone $(P=0.02)$.

\section{Influence of the cost of EUS-FNA + nCLE}

As described earlier, the cost of nCLE is estimated at $600 €$ (cost estimate provided by Mauna Kea Technologies). Since this is an estimation, calculations were also performed using a cost range between $300 €$ and $900 €$ : the overall benefit was maintained even using the higher cost estimate of $900 €$ (total cost using EUS-FNA + nCLE of $7387000 €$ and $4023000 €$ based on public and private sector rates compared to, respectively, $8111012.95 €$ and $4310718.42 €$ for EUS-FNA alone [see $>$ Table 4]). This represents a cost reduction of approximately $9 \%$ and $7 \%$ for the public and private sectors, respectively, even using the higher cost estimate of $900 €$ for nCLE.

\section{Stochastic sensitivity analysis: performance of tests}

A total of 100 simulations were performed, each one using different sensitivity and specificity values taken randomly from the ranges described above ( $\mathbf{F i g . 3}$ ). In each case, the number of interventions was less for EUS-FNA + nCLE than for EUS-FNA alone, and the highest number of interventions for EUS-FNA+ nCLE (395) was less than the lowest number of interventions for EUS-FNA alone (493). The distribution of the number of interventions for EUS-FNA + nCLE had a mean value significantly lower than that for EUS-FNA alone $(P<0.00001)$ for the 100 simulations.

\section{Discussion}

The limited diagnostic performance of EUS-FNA alone for pancreatic cyst characterization, especially for SCAs, can result in a high rate of diagnostic uncertainties, leading to unnecessary surgery, long-term follow-up and surveillance, and patient stress. This leads to a significant healthcare cost that could be avoided by improving the diagnosis of such cysts. Even with no surgical intervention, the cost of life-long surveillance of benign cysts is significant, and causes unnecessary anxiety to the patient.

The use of EUS-FNA + nCLE improves the sensitivity and specificity of the diagnostic technique, allowing a reduction in diagnosis doubt and a much improved rate of benign cyst (SCA+) identification, i.e. those that do not require surgical intervention or intensive follow-up. Although the diagnosis of pancreatic malignancies has improved in recent decades, with a reduction in pancreatic resection in those diagnosed from $94 \%$ in 1990 to $54 \%$ in 2014 [9], there remains diagnostic doubt. The elimination of uncertainties by using EUS-FNA + nCLE can further improve diagnostic accuracy and significantly reduce the number of surgical interventions $(P=0.02)$. A recent large-scale trial has been conducted in more than 200 patients scheduled for assessment by EUS-FNA for a large $(\geq 2 \mathrm{~cm})$ solitary pancreatic cystic lesion (PCL) without evidence of communication with the main pancreatic duct and without evidence of malignancy or chronic pancreatitis. Preliminary results confirm the very high sensitivity and specificity of nCLE criteria for the most fre-

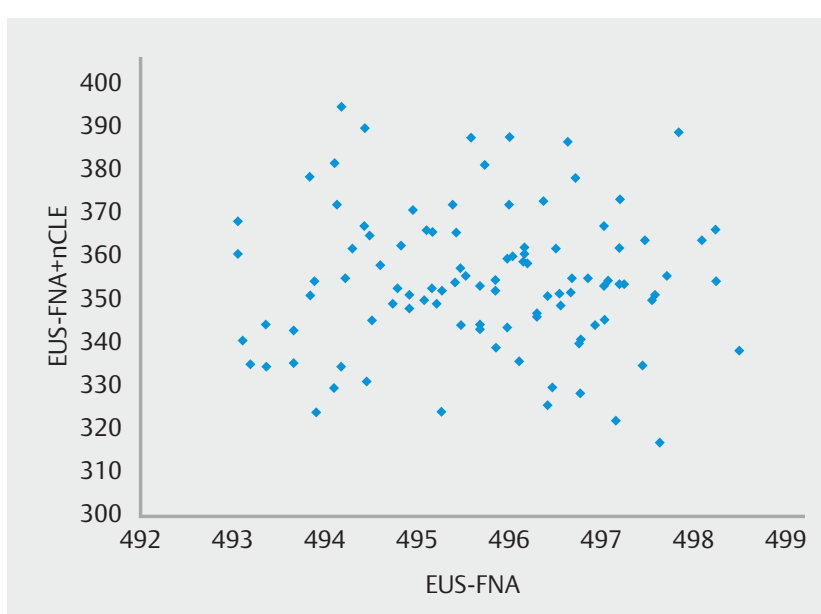

- Fig. 3 Stochastic sensitivity analysis for test performance. (Note: each point corresponds to one simulation of the number of surgical interventions resulting from each diagnostic procedure, using sensitivity and specificity ranges described in $>$ Table 3 .)

quently observed PCLs [21]. The addition of nCLE to the EUSFNA procedure could now systematically be considered in this group of patients, having been shown to positively impact patient management.

Using probability calculations based on historical data from 1990 to 2014, our study shows that the addition of nCLE to EUS-FNA in a hypothetical cohort of 1000 patients would provide an economic benefit by improving the accuracy of diagnosis, better identifying patients at risk of pancreatic malignancy, and so reducing the number of surgical interventions. Additionally, overall the lives of 4 in 1000 patients would be saved by using EUS-FNA + nCLE due to the elimination of mortality associated with unnecessary surgery.

It should be noted that, as all parameters are modeled (and not outputs from studies), the number of patients included ( $n=$ 1000 ) is simply a neutral scaling factor: any other number would have led to results that would be different only by a multiplicative factor.

Extensive sensitivity and specificity analyses were performed to assess the robustness of the findings, and the sensitivity of the model to variations in parameters was measured. An evaluation of the influence of the a priori probability, of the surgical rules in the EUS-FNA rules, and of the cost of the EUS$\mathrm{FNA}+\mathrm{nCLE}$ procedure strengthened the model by showing that variations in these parameters do not change the overall conclusions with regard to the benefits of EUS-FNA + nCLE compared to EUS-FNA.

Of note is that this economic benefit takes into account only the diagnostic procedure itself, the associated reduced rate of surgery, and the related costs associated with clinical intervention and hospital stay. The real cost benefit would be significantly higher: better detection of true SCA+ using EUS-FNA+ nCLE would logically reduce the number of repeated diagnostic procedures and the number of subsequent surveillance examinations, as well as resulting in fewer re-hospitalizations and less long-term treatment required following DP or LP. 
Large differences between the private and public sectors have two explanations. First, in the public sector, the cost of stay includes the physicians' salaries (surgeons and anesthesiologists) whereas in the private sector, physicians are independent and bill the fees directly to patients in addition to the cost of the stay. Second, the proportion of DP and LP differs between sectors: the relative proportions of DP/LP are 80/20 in the public sector and 50/50 in the private sector, the cost of DP being approximately twice as much as LP (1021.74€ vs. $540.05 €$, excluding anesthesiology). Even after adjusting for these two factors, differences remain for institutional reasons (e. g. some public hospitals are university hospitals, which include an emergency service; this is not the case for all private hospitals).

As well as a reduced rate of surgical intervention, this study also demonstrates a slight reduction in "false negatives" using nCLE. Although slight, this represents a further reduction in morbidity and mortality and a further improvement in the medico-economic benefits of EUS-FNA + $\mathrm{nCLE}$.

A limitation of this study is that the costs given are valid only for France. Due to national differences in healthcare systems and costs, no economic evaluation can be universally valid and the results necessarily depend on a specific national context. The economic benefit of EUS-FNA+ $\mathrm{nCLE}$ in other countries could be assessed using local data in the model structure to reflect national specificities for healthcare reimbursement and patient management for pancreatic cysts. Another limitation of the study is that other methods for the diagnosis of SCA such as CEA (using $5 \mathrm{ng} / \mathrm{mL}$ as a cutoff) and cyst morphology observed during imaging (apart from communication) are not taken into account in the medico-economical model. However, the limits of these have been described by Jais et al. [9] and confirmed in a recent multicenter study in 209 patients with large non-communicating pancreatic cysts [21] which showed the accuracy of CEA $<5 \mathrm{ng} / \mathrm{mL}$ and $\mathrm{nCLE}$ for the diagnosis of benign cystic lesions to be $84 \%$ and $96 \%$, respectively.

In conclusion, the adoption of routine nCLE in addition to EUS-FNA alone for the diagnosis of isolated, non-communicating cysts without CCP would lead to significant economic benefits to healthcare systems by reducing the number of "false positives" as well as "false negatives" due to improved diagnostic accuracy.

\section{Acknowledgments}

The authors thank Andrew Lane (Lane Medical Writing) who provided professional medical writing assistance (funded by Mauna Kea Technologies) in accordance with the European Medical Writers Association guidelines and Good Publication Practice, and Claire Burucoa, Nathalie Chabin, and Aline Criton from Mauna Kea Technologies for their valuable input and support in this project.

This study was supported by Mauna Kea Technologies, Paris, France.

\section{Competing interests}

Claude Le Pen designed and implemented the economic model and received consultancy fees from Mauna Kea Technologies.

\section{References}

[1] de Jong K, Bruno MJ, Fockens P. Epidemiology, diagnosis, and management of cystic lesions of the pancreas. Gastroenterol Res Pract 2012; 2012: 147465

[2] de Jong K, Nio CY, Hermans JJ et al. High prevalence of pancreatic cysts detected by screening magnetic resonance imaging examinations. Clin Gastroenterol Hepatol 2010; 8: 806-811

[3] de Jong K, Nio CY, Mearadji B et al. Disappointing interobserver agreement among radiologists for a classifying diagnosis of pancreatic cysts using magnetic resonance imaging. Pancreas 2012; 41 : $278-282$

[4] Park WG, Mascarenhas R, Palaez-Luna M et al. Diagnostic performance of cyst fluid carcinoembryonic antigen and amylase in histologically confirmed pancreatic cysts. Pancreas 2011; 40: $42-45$

[5] Nakai Y, Iwashita T, Shinoura $S$ et al. Role of serial EUS-guided FNA on pancreatic cystic neoplasms: a retrospective analysis of repeat carcinoembryonic antigen measurements. Gastrointest Endosc 2016; 84: $780-784$

[6] Napoleon B, Lemaistre AI, Pujol B et al. A novel approach to the diagnosis of pancreatic serous cystadenoma: needle-based confocal laser endomicroscopy. Endoscopy 2015; 47: 26-32

[7] Napoleon B, Lemaistre Al, Pujol B et al. In vivo characterization of pancreatic cystic lesions by needle-based confocal laser endomicroscopy (nCLE): proposition of a comprehensive $\mathrm{nCLE}$ classification confirmed by an external retrospective evaluation. Surg Endosc 2016; 30: $2603-2612$

[8] Bruno M]. Novel diagnostic approach to pancreatic cysts: is confocal laser endomicroscopy bridging the gap? Endoscopy 2015; 47: 4-5

[9] Jais B, Rebours V, Malleo G et al. Serous cystic neoplasm of the pancreas: a multinational study of 2622 patients under the auspices of the International Association of Pancreatology and European Pancreatic Club (European Study Group on Cystic Tumors of the Pancreas). Gut 2016; 65: 305-312

[10] Brugge WR, Lauwers GY, Sahani D et al. Cystic neoplasms of the pancreas. NEJM 2004; 351: $1218-1226$

[11] Belsley NA, Pitman MB, Lauwers GY et al. Serous cystadenoma of the pancreas: limitations and pitfalls of endoscopic ultrasound-guided fine-needle aspiration biopsy. Cancer 2008; 114: 102 - 110

[12] Le Borgne J, de Calan L, Partensky C. Cystadenomas and cystadenocarcinomas of the pancreas: a multiinstitutional retrospective study of 398 cases. French Surgical Association. Ann Surg 1999; 230: 152 161

[13] Maker AV, Lee LS, Raut CP et al. Cytology from pancreatic cysts has marginal utility in surgical decision-making. Ann Surg Oncol 2008; 15: 3187-3192

[14] Müssle B, Distler M, Wolk S et al. Management of patients with pancreatic cystic lesions: a case-based survey. Pancreatology 2017; 17: $431-437$

[15] Thornton GD, McPhail M], Nayagam S et al. Endoscopic ultrasound guided fine needle aspiration for the diagnosis of pancreatic cystic neoplasms: a meta-analysis. Pancreatology 2013; 13: 48-57

[16] Thosani N, Thosani S, Qiao W et al. Role of EUS-FNA-based cytology in the diagnosis of mucinous pancreatic cystic lesions: a systematic review and meta-analysis. Dig Dis Sci 2010; 55: 2756 - 2766 
[17] Tseng JF, Warshaw AL, Sahani DV et al. Serous cystadenoma of the pancreas: tumor growth rates and recommendations for treatment. Ann Surg 2005; 242: $413-419$

[18] Fernandez-del Castillo C, Targarona J, Thayer SP et al. Incidental pancreatic cysts: clinicopathologic characteristics and comparison with symptomatic patients. Arch Surg 2003; 138: 427-433

[19] Agence Technique de l'Information su l'Hospitalisation. Available from: www.atih.sante.fr/ (Accessed: 12 September 2017 [in French])
[20] Vege SS, Ziring B, Jain R et al. American gastroenterological association institute guideline on the diagnosis and management of asymptomatic neoplastic pancreatic cysts. Gastroenterology 2015; 148: $819-822$

[21] Napoleon B, Pujol B, Palazzo M et al. Needle-based confocal laser endomicroscopy (nCLE) for the diagnosis of pancreatic cystic lesions: preliminary results of the first prospective multicenter study (Abstract 602). Gastroenterology 2017; 152: S132-S133 